\title{
24. Landwirtschaft und Medizin - Antipoden bei der Wahrnehmung der Gentechnik in Deutschland
}

\subsection{Einleitung}

Neben der Kernenergie gehört die Gentechnik zu den technischen Innovationen, die in Deutschland - und nicht nur hier - die intensivsten gesellschaftlichen Kontroversen ausgelöst haben. Dabei gibt es, wie empirische Studien bereits in den 1990er Jahren gezeigt haben, keine generelle Ablehnung der Gentechnik, sondern stark variierende Bewertungen konkreter Anwendungen der Gentechnik, die von allgemeiner Zustimmung bis hin zu verbreiteter Ablehnung reichen (vgl. Hampel/Renn, 1999). Grob gesagt kann zwischen einer bis heute weitgehend abgelehnten Anwendung der Gentechnik in der Landwirtschaft und einer weitgehend akzeptierten Anwendung der Gentechnik in der Medizin unterschieden werden.

Einen Höhepunkt erreichten die Debatten um die Gentechnik in den späten 1990er Jahren, den „years of controversy“ (Durant et al., 2001), in denen sowohl der Import genetisch veränderten Sojas, das Klonschaf Dolly und nach der Jahrtausendwende die Frage der Forschung mit menschlichen embryonalen Stammzellen für intensive Diskussionen sorgten (vgl. u. a. Torgersen et al., 2002).

Dass sich diese Debatten sukzessiv beruhigt haben, lag daran, dass zum einen die Stammzelldebatte politisch gelöst wurde und sich zum anderen die in der Öffentlichkeit weitgehend abgelehnte Grüne Gentechnik aus unterschiedlichen Gründen nicht durchsetzen konnte (siehe auch van den Daele/Broer, Kap. 21, und Dederer, Kap. 22). Mangels konkreten, als Gentechnik identifizierbaren Objekten ist die Grüne Gentechnik aus dem Fokus gesellschaftlicher Debatten verschwunden. Mit neuen, präziseren Verfahren genetischer Veränderungen (Genome-Editing), insbesondere mittels 
der Genschere CRISPR/Cas9, ${ }^{1}$ erfährt der Disput eine Wiederbelebung (siehe Clemens, Kap. 7). Diskutiert werden insbesondere die Möglichkeiten und Grenzen der Gentherapie (vgl. Reich et al., 2015; siehe auch Fehse, Kap. 6), aber auch die grundsätzliche Frage, ob es sich bei den neuen Züchtungsformen um Gentechnik handelt oder nicht (siehe auch Fehse et al., Kap. 9).

Die Beruhigung der gesellschaftlichen Auseinandersetzung um die Gentechnik nach den „years of controversy“ (Gaskell/Bauer, 2001) ging auch mit einer deutlichen Verschlechterung der Datenlage zu Einstellungen der deutschen Bevölkerung zur Gentechnik einher. Bis 2010 gab es regelmäßige Befragungen zu Einstellungen zur Gentechnik, insbesondere durch einschlägige Eurobarometer-Befragungen der EU-Kommission, die zwischen 1991 und 2010 durchgeführt wurden und vergleichbare Daten bereitgestellt haben. Seither ist ein Mangel an qualitativ guten wissenschaftlichen Daten zur Haltung der deutschen Öffentlichkeit gegenüber der Gentechnik zu beklagen (vgl. Hampel/ Zwick, 2016). Dies ist umso bedauerlicher, als in den letzten Jahren das Innovationstempo in diesem Technologiesegment zugenommen hat und nicht zuletzt dank CRISPR/ Cas9 eine Vielzahl neuer Anwendungen diskutiert werden (siehe Clemens, Kap. 7).

\subsection{Das TechnikRadar}

Um dem Defizit an belastbaren wissenschaftlichen Daten zur Wahrnehmung und Bewertung moderner Technologien, nicht nur zur Gentechnik, abzuhelfen, wurde das TechnikRadar von acatech - der Deutschen Akademie der Technikwissenschaften und der Körber-Stiftung ins Leben gerufen. Konzipiert und wissenschaftlich ausgewertet wird es vom Zentrum für Interdisziplinäre Risiko- und Innovationsforschung der Universität Stuttgart (ZIRIUS). Die jährliche Analyse bietet fundierte Impulse für die öffentliche Debatte um den Stellenwert, die Gestaltung und die Regulierung technischer Innovationen.

Dazu werden im zweijährigen Turnus bundesweite Repräsentativbefragungen (CATI: Computer Assisted Telephone Interview) mit jeweils 2.000 Befragten durchgeführt. Ergänzt werden diese quantitativen Erhebungen durch qualitative Studien, die

1 CRISPR/Cas9 ist eine Methode zur gentechnischen Veränderung von Organismen. Das Akronym CRISPR steht dabei für Clustered Regularly Interspaced Short Palindromic Repeats und bezeichnet ursprünglich bestimmte Sequenzen im Genom von Bakterien, die mit einer spezifischen Immunantwort gegen Viren zusammenhängen. Cas9 ist ein mit diesen Sequenzen assoziiertes Protein, welches das virale Erbgut schneiden kann. 2012 wurde veröffentlicht, dass Cas9 im Labor so programmiert werden kann, dass es spezifisch vorgegebene Sequenzen im Genom finden und schneiden kann (Jinek et al., 2012). Seither wurde die Methode fortlaufend weiterentwickelt. 
es erlauben, die Hintergründe der Technikwahrnehmung zu erkunden. Mit dem TechnikRadar 2020 (acatech/Körber-Stiftung, 2020) sind zum ersten Mal seit längerer Zeit Einstellungsdaten zur Gentechnik verfügbar, die mit einem wissenschaftlichen Interesse und einer an den Standards der empirischen Sozialforschung orientierten Stichprobe erhoben wurden.

Das TechnikRadar 2020 mit seinem Schwerpunkt Bioökonomie enthält unter anderem Fragen zur Anwendung der Gentechnik in der Landwirtschaft, zu Kunstfleisch, zu Ersatzorganen (aus Stammzellen und von Xenotransplantationen) und zur Gentherapie. In allen drei Anwendungsbereichen wurden zu Vergleichszwecken Einstellungen zu verschiedenen, teilweise auch nicht-gentechnischen Verfahren erhoben. Die wissenschaftliche Bearbeitung des TechnikRadar 2020 oblag dem Autorenteam dieses Beitrags.

\subsection{Gentechnik in Landwirtschaft und Ernährung}

Zahlreiche empirische Studien haben gezeigt, ${ }^{2}$ dass die Grüne Gentechnik von großen Teilen der Öffentlichkeit abgelehnt wird. Betrachtet man empirische Studien, insbesondere die einschlägigen Eurobarometer-Untersuchungen, kann hierfür, anders als gelegentlich unterstellt, weder eine besondere Risikowahrnehmung ihrer Anwendungen noch eine besondere Risikoaversion verantwortlich gemacht werden. Die breite Ablehnung der Grünen Gentechnik fußt vielmehr auf fehlender Nutzenwahrnehmung (Gaskell et al., 2004) und ihrer Inkompatibilität mit Werten von ,Natürlichkeit' und ,Tradition', die bis heute von der Werbewirtschaft bedient und verstärkt werden. Auf den europäischen Lebensmittelmärkten spielen gentechnisch veränderte Lebensmittel, soweit sie kennzeichnungspflichtig sind, daher keine Rolle.

Mit der Einführung neuer Züchtungsverfahren durch CRISPR/Cas9 ist die Grüne Gentechnik noch einmal auf die Agenda gekommen, da die neuen Möglichkeiten einerseits die gentechnische Bearbeitung neuer Pflanzen erleichtern und beschleunigen, andererseits neben transgenen Pflanzen ${ }^{3}$ auch solche Pflanzen erzeugt werden können, die nur minimale Änderungen an einzelnen Basen im Genom aufweisen und die keine artfremde DNA enthalten (siehe Clemens, Kap. 7). Aufgrund der Tatsache, dass einzelne Mutationen auch natürlich vorkommen könnten, stellte sich dabei die Frage, ob Letztere als gentechnisch veränderte Pflanzen unter das Gentechnikrecht fallen oder nicht. Dem Urteil des Europäischen Gerichtshofes (EuGH) zur Gentechnik und Mutagenese-

2 Eine Übersicht findet sich bei Hampel/Zwick (2016).

3 Transgene Pflanzen enthalten genetisches Material anderer Arten, etwa aus Bakterien. 
züchtung von $2018^{4}$ zufolge fällt die Pflanzenzüchtung mit CRISPR/Cas9 unter das Gentechnikrecht - im Gegensatz zur Mutagenesezüchtung, bei der durch Strahlung oder chemische Einflüsse Mutationen ausgelöst werden (siehe hierzu Dederer, Kap. 22). Diese Züchtungsform wurde zwar als Gentechnik eingestuft, jedoch von der Regulierung ausgenommen, da es sich um eine seit Jahrzehnten etablierte und als sicher geltende Methode handelt.

Im TechnikRadar 2020 haben wir die Einstellungen zu beiden Züchtungstechniken klassischer Gentechnik, also der gezielten genetischen Veränderung von Nutzpflanzen, und Mutagenese, dem ungezielten Auslösen von genetischen Veränderungen in Nutzpflanzen durch Bestrahlung oder chemische Einwirkungen - untersucht. Zur Mutagenesezüchtung, einer Züchtungsform, die immerhin seit einigen Jahrzehnten angewandt wird, liegen mit dem TechnikRadar erstmals Daten vor. ${ }^{5}$ Die bisherige Nichtbeachtung der Mutagenesezüchtung in der sozialwissenschaftlichen Einstellungsforschung ist vermutlich darauf zurückzuführen, dass sie in der Vergangenheit wenig umstritten war. Mit dem TechnikRadar haben wir hier diesbezüglich Neuland betreten.

Vor dem Hintergrund, dass die Grüne Gentechnik anders als die Mutagenesezüchtung ${ }^{6}$ seit Jahrzehnten eine hochkontroverse Technologie ist, wäre zu erwarten, dass sich die Einstellungen zu beiden Züchtungstechnologien grundlegend unterscheiden. Die Ergebnisse des TechnikRadar zeigen aber etwas anderes (vgl. Abb. 1): Wie erwartet wird die Grüne Gentechnik weitgehend abgelehnt. Nur rund jeder Siebte befürwortet sie, etwas mehr als die Hälfte (57,5 \%) lehnt sie ab. Wenn man bedenkt, dass die gentechnische Pflanzenzucht Gegenstand intensiver gesellschaftlicher Kontroversen und politischer Regulierungsaktivitäten war und ist, während die Mutagenesezüchtung nie problematisiert wurde, überrascht, dass Letztere auf noch stärkere Ablehnung (61,1 \%)

4 C-528/16, siehe unter: https://eur-lex.europa.eu/legal-content/DE/TXT/PDF/?uri=CELEX: 62016CJ0528\&from=DE [17.03.2021].

5 Im Fragebogen wurde Mutagenese beschrieben als das „Auslösen ungezielter genetischer Veränderung von Saatgut durch Bestrahlung oder Chemikalien, um neue Sorten zu erhalten. Hieraus können Pflanzen mit gewünschten Eigenschaften ausgewählt werden“. Grüne Gentechnik wurde definiert als „gentechnischer Eingriff. D. h. Gene werden gezielt eingefügt, ausgeschaltet oder verändert, um Pflanzen mit gewünschten Eigenschaften zu erhalten." Auf einen direkten Verweis auf CRISPR/Cas9 wurde verzichtet, da nicht davon ausgegangen werden konnte, dass die neuen Verfahren allgemein bekannt sind. Aus Untersuchungen zur Wahrnehmung von cisgenen Pflanzen, denen genetisches Material derselben Art zugefügt wurde, und transgenen Pflanzen ist bekannt, dass weniger die Art der Veränderung als die Tatsache, ob überhaupt eine beabsichtigte genetische Veränderung vorgenommen wird, für die Einstellungsbildung zentral ist (Kronberger et al., 2013).

6 Aus Kapazitätsgründen konnte nicht untersucht werden, ob den Befragten bewusst war, dass zahlreiche Nutzpflanzen seit Langem auf der Mutagenesezüchtung beruhen. 
stößt als Gentechnik in der Pflanzenzucht und auf noch geringere Zustimmung. Beide Technologien werden in der Wahrnehmung der Öffentlichkeit sehr ähnlich beurteilt $\left(\mathrm{r}=0,62^{* *}\right) .^{7}$

Abbildung 1: Einstellungen zu Gentechnik und Mutagenesezüchtung; in \%

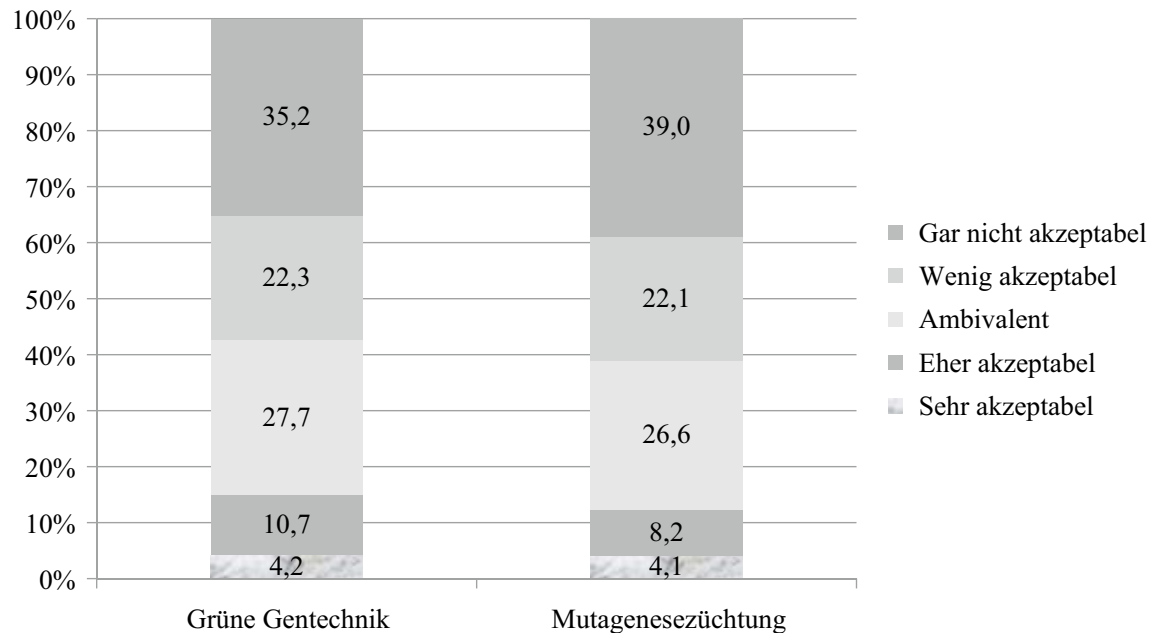

Datenbasis: TechnikRadar 2020, N=2006.

Dieses, in Anbetracht der höchst unterschiedlichen gesellschaftlichen Reaktionen auf beide Technologien, überraschende Ergebnis ist vermutlich darauf zurückzuführen, dass in Deutschland den Werten Natur und Natürlichkeit ein sehr hoher Stellenwert eingeräumt wird. So hält die Mehrheit (50,7 \%) der Befragten des TechnikRadar „die konsequente Verwertung der Natur durch den Menschen“ für mehr oder weniger verwerflich. Auch ein Verfahren wie die Mutagenesezüchtung, bei der Saatgut durch technische Eingriffe zu Mutationen angeregt wird, scheint nicht mit den romantisierenden Vorstellungen von einer natürlichen Landwirtschaft vereinbar zu sein.

7 Pearsons $r$ wird eingesetzt, um bei metrisch skalierten Variablen zu messen, wie stark y mit ansteigendem $\mathrm{x}$ linear zu- (positives $\mathrm{r}$ ) oder abnimmt (negatives $\mathrm{r}$ ). Metrisch skaliert sind Variablen, bei denen die Abstände zwischen den einzelnen Merkmalsausprägungen jeweils identisch sind. Pearsons $r$ kann Werte zwischen -1 und +1 annehmen. Den Wert , 0 nimmt Pearsons $r$ an, wenn es zwischen zwei Variablen keinen Zusammenhang gibt. Bei einem perfekten negativen Zusammenhang zwischen zwei Variablen erhalten wir einen r-Wert von -1, bei einem perfekten positiven Zusammenhang erhalten wir den +1 . Ein perfekter Zusammenhang besteht dann, wenn die abhängige Variable vollständig durch die unabhängige Variable statistisch erklärt werden kann. Pearsons r erlaubt es, den durch die unabhängige Variable erklärten Anteil der Varianz der abhängigen Variable zu berechnen. 
Auffällig ist, dass es in Bezug auf die Einstellungen zu Grüner Gentechnik und Mutagenesezüchtung nur geringe soziodemographische Unterschiede gibt. Ebenso wenig unterscheidet sich die Zustimmung zu Grüner Gentechnik und Mutagenesezüchtung bei Personen mit unterschiedlichen Bildungsniveaus $(\gamma=-0,07) .{ }^{8}$ Ein bei zahlreichen Untersuchungen zu Technikeinstellungen gefundenes Muster finden wir auch bei den Einstellungen zur Grünen Gentechnik. Besonders kritisch sind ältere Frauen (65 und älter), besonders aufgeschlossen junge Männer (unter 35). Während gut ein Viertel der jungen Männer Gentechnik in der Pflanzenzucht für akzeptabel hält (27,7 \%), ist es bei den älteren Frauen nur jede Neunte (11,3 \%). Ein ähnliches Muster, wenn auch nicht so ausgeprägt, finden wir auch bei den Einstellungen zur Mutagenesezüchtung, wobei hier die Ablehnung bei Frauen im mittleren Alter (35-65) besonders ausgeprägt ist (73,7 \%).

Die Daten des TechnikRadar verdeutlichen, dass die Grüne Gentechnik als Risikotechnologie wahrgenommen wird: zwei Drittel der Deutschen $(66,4 \%)$ halten sie für eher oder sehr riskant. Für eine Methode zur Lebensmittelherstellung ist es hoch problematisch, dass nur jeder Neunte (11,6 \%) sie für wenig oder gar nicht riskant hält. Bedeutsam ist auch, dass aus Sicht der Öffentlichkeit wenig dafür spricht, die wahrgenommenen Risiken in Kauf zu nehmen. Nur von einer Minderheit (20,9 \%) wird die Grüne Gentechnik als nützlich wahrgenommen; ein Ergebnis, das auch aus früheren Untersuchungen bekannt ist. ${ }^{9}$ Dabei sind Nutzen- und Risikowahrnehmung stark negativ korreliert $\left(\gamma=-0,55^{* *}\right):{ }^{10}$ je größer die Risikowahrnehmung, desto geringer die Nutzenwahrnehmung und vice versa.

Obwohl die festgestellte verbreitete Ablehnung der Mutagenese durchaus ein Potenzial für Kontroversen signalisiert, ist dies aus mehreren Gründen unwahrscheinlich: Zum einen wird die Mutagenese als Züchtungsform seit langem praktiziert und hat sich in dieser Zeit als unproblematisch erwiesen. ${ }^{11}$ Zum anderen ist es unwahrscheinlich, dass etwa Umweltgruppen diese Anwendung, die bereits erfolgte, bevor die meisten

$8 \gamma$ wird eingesetzt, um bei ordinal skalierten Variablen, also Variablen, deren Ausprägungen zwar eine Rangordnung erlauben, deren Abstände aber unbestimmt sind, zu messen, wie stark y bei ansteigendem x zunimmt (positives $\gamma$ ) oder abnimmt (negatives $\gamma$ ). $\gamma$ kann wie $r$ Werte zwischen -1 und +1 annehmen. $\gamma$ kann ähnlich wie $\mathrm{r}$ interpretiert werden. Es ist allerdings nicht möglich, den statistisch erklärten Varianzanteil zu berechnen.

9 Vgl. zur Bedeutung von Nutzen- und Risikowahrnehmung Gaskell et al. (2004).

10 ** bedeutet, dass der Zusammenhang auf dem $1 \%$ Niveau statistisch signifikant ist. Das bedeutet, dass die Wahrscheinlichkeit, dass der Zusammenhang zwischen beiden Variablen nur zufällig ist, bei unter $1 \%$ liegt. * bedeutet entsprechend, dass der Zusammenhang auf dem 5 \% Niveau statistisch signifikant ist.

11 In diese Richtung argumentiert auch der EuGH bei seinem Urteil zu CRISPR/Cas9. 
Umweltverbände gegründet wurden, nach so langer Zeit der nicht problematisierten Anwendung noch einmal als Thema aufgreifen werden.

\subsection{Laborfleisch - eine mögliche Alternative?}

Nicht nur die Grüne Gentechnik wird kontrovers diskutiert, auch Fleischkonsum und Fleischproduktion geraten zunehmend unter gesellschaftlichen Druck. Kritisiert werden vor allem ökologische Auswirkungen wie etwa der Flächenverbrauch für die Futtermittelproduktion oder $\mathrm{CO}_{2}$-Emissionen sowie tierethische Probleme, allen voran Massentierhaltung und Schlachtung. Seit Jahren wird in der Wissenschaft an alternativen Fleischangeboten, wie beispielsweise In-vitro- bzw. Kunstfleisch gearbeitet. Dazu werden aus den Muskeln von Tieren Stammzellen entnommen, aus denen mithilfe einer Nährlösung künstliches Fleisch produziert wird; Massentierhaltung und massenhafte Schlachtungen würden damit entfallen. Aus ethischer Perspektive problematisch ist die Verwendung fetalen Kälberserums als Nährmedium, für das aber mittlerweile an Alternativen gearbeitet wird (Woll, 2019): Für die Befragung vom TechnikRadar wurde Laborfleisch mit der Option von Algenextrakt als Nährlösung abgefragt. Die Herstellung von Kunstfleisch ist bereits gelungen (Post, 2014); in einigen Jahren wird mit der Marktreife und konkurrenzfähigen Produkten gerechnet, womit die Frage, auf welche Resonanz Laborfleisch stößt, zunehmend an Bedeutung gewinnt.

In einer Eurobarometer-Befragung aus dem Jahre 2005 wurde unter dem Aspekt von genetisch veränderten Organismen nach Fleisch aus dem Labor gefragt: Selbst unter der Bedingung, dass dadurch keine Tiere mehr geschlachtet werden müssten, überwog die Ablehnung, wobei sich die Deutschen nur geringfügig vom europäischen Durchschnitt unterscheiden (58 \% gegenüber 54 \% im EU-Durchschnitt) (vgl. EU, 2005: 92).

Eine aktuelle Literaturübersicht von Bryant und Barnett (2018) kommt ebenfalls zu dem Ergebnis, dass die Mehrheit der Verbraucher kultiviertes Fleisch („cultured meat“) ablehnt. Nur eine Minderheit würde das Laborfleisch herkömmlichem Fleisch vorziehen. Gründe für die Ablehnung waren demnach insbesondere die Unnatürlichkeit von Laborfleisch, Sicherheitsbedenken, Geschmack und Preis, aber auch Auswirkungen auf die traditionelle Landwirtschaft sowie Misstrauen gegenüber den Laborfleisch produzierenden Konzernen (Bryant/Barnett, 2018: 12 ff.). Der Nutzen von Laborfleisch wurde vor allem hinsichtlich Umweltauswirkungen und beim Tierwohl gesehen.

Das TechnikRadar 2020 gelangt zu ähnlichen Ergebnissen. Die deutsche Öffentlichkeit kommt mehrheitlich (63,6 \%) ebenfalls zu einer negativen Einschätzung von Laborfleisch und nur 15,7 \% teilen die Auffassung, Laborfleisch sei „eine gute Sache“. Wie bereits bei der Grünen Gentechnik und bei der Mutagenesezüchtung äußern sich Frauen 
kritischer als Männer, dies gilt insbesondere für weibliche Befragte mittleren $(74,4 \%)$ und höheren Lebensalters (76,3 \%), die Kunstfleisch ablehnen. Auf mehr Sympathie stößt Fleisch aus dem Labor vor allem bei jüngeren Personen, wobei 31,8 \% der jungen Männer und 25,2 \% junger Frauen diese Produkte aufgeschlossen beurteilen $\left(C_{\text {korr }}=0,33^{* *}\right) .{ }^{12}$

Die überwiegend negative Einschätzung liegt nicht zuletzt daran, dass die Gründe für Laborfleisch, die Innovatoren und Protagonisten zumeist aus dem Forschungs- und aus dem Start-up-Bereich anführen, nicht akzeptiert werden: umweltverträglicher, tierfreundlicher, gesünder, sicherer sowie ein möglicher Lösungsansatz zur Bewältigung der globalen Ernährungsproblematik (vgl. bspw. Böhm et al., 2018). Für knapp die Hälfte der Deutschen (47,1 \%) tragen vermutete Risiken zur Ablehnung von Laborfleisch bei. Dass Laborfleisch eine ethisch vertretbare Alternative zu konventionellem Fleisch sei, wird ebenfalls mehrheitlich (55,2\%) abgelehnt (vgl. zur Wahrnehmung ethischer Aspekte auch Böhm et al., 2018; Laestadius, 2015; vgl. zur Diskussion ethischer Aspekte Woll, 2019). Über zwei Drittel der Deutschen (64,6 \%) sehen durch Fleisch, das im Labor erzeugt wird, eine weitere Entfremdung der Menschen von der Erzeugung ihrer Nahrungsmittel.

Davon, dass Laborfleisch eine ethisch verträgliche Alternative sei, sind vor allem die Jüngeren und die besser Gebildeten überzeugt (44,6\%). Dagegen teilt nur knapp jeder Elfte $(8,9 \%)$ der Befragten, die mindestens 65 Jahre alt sind und maximal die Mittlere Reife haben, diese Einschätzung $\left(\mathrm{C}_{\mathrm{korr}}=0,32^{* *}\right)$.

Dass man mit Laborfleisch die globalen Ernährungsprobleme lösen könne, erwarten ebenfalls vor allem jüngere, männliche Befragte (38,5\%), aber beispielsweise nur 12,6 \% der über 65-jährigen Frauen $\left(C_{\text {korr }}=0,30^{* *}\right)$.

Insgesamt sind, das zeigen die Resultate des TechnikRadar, noch am ehesten die Jüngeren und hier vor allem die jungen Männer gegenüber dieser Anwendung aufgeschlossen. Es stellt sich die Frage, ob wir es hier mit einem Alters- oder mit einem Kohorteneffekt zu tun haben. Bei einem Kohorteneffekt wäre zu erwarten, dass die jetzt gegenüber Laborfleisch aufgeschlossenen Jüngeren auch in Zukunft aufgeschlossener bleiben. Bei einem Alterseffekt wäre zu erwarten, dass die jetzt Aufgeschlossenen im Laufe ihres Lebens kritischer werden und sich nach einer Phase jugendlicher Aufgeschlossenheit in ihrem späteren Urteil den gegenwärtig älteren Befragten angleichen.

12 Der korrigierte, $d$. h. auf einen Wertebereich von 0 ...1 normierte Kontingenzkoeffizient $\left(\mathrm{C}_{\text {korr }}\right)$ misst die Stärke des Zusammenhangs von nominal skalierten Variablen. Der Wert , 0 ‘ bedeutet, dass es zwischen zwei Variablen keinen Zusammenhang gibt, der Wert , $1^{\prime}$, dass es einen perfekten Zusammenhang zwischen zwei Variablen gibt, d. h., dass ein Wert der einen Variable immer und genau immer mit einem bestimmten Wert der anderen Variablen einhergeht. 
Einschlägige Untersuchungen zur Grünen Gentechnik ${ }^{13}$ zeigen, dass das Phänomen der gegenüber dieser Technologie aufgeschlosseneren jüngeren Männer immer wieder zu beobachten ist, nicht aber eine Veränderung der Einstellungen der mittleren und älteren Alterskohorten - Befunde, die klar zugunsten eines Alterseffekts sprechen.

\subsection{Medizin}

\subsubsection{Ersatzorgane}

Seit mehr als sechs Jahrzehnten zählen Organtransplantationen zum Repertoire medizinischer Leistungen und an entsprechenden Kliniken beinahe schon zu den Routineeingriffen im klinischen Alltag. Der Erfolg von Organtransplantationen wird allerdings von einem Mangel an Spenderorganen begleitet. Auf der Suche nach Alternativen zu menschlichen Spenderorganen werden gegenwärtig zwei technische Lösungen diskutiert: Die Herstellung von Organen aus Stammzellen und Xenotransplantationen (De Cheveigné et al., 2006).

Bei Xenotransplantationen werden die Tiere genetisch so verändert, dass ihre Organe als Spenderorgane für Menschen in Frage kommen. Anstrengungen in diese Richtung werden bereits seit dem Ende der 1990er Jahre intensiv diskutiert (Bundesärztekammer, 1999; Petermann/Sauter, 1999; Schicktanz, 2000), auch wenn die öffentliche Aufmerksamkeit für diese Forschungen anfänglich - nicht nur in Deutschland - nur sehr gering war (De Cheveigné et al., 2006). Hat die Kontroverse um die Grüne Gentechnik, die Anwendung der Gentechnik in der Landwirtschaft, eine breite Öffentlichkeit erreicht, werden Probleme medizinischer Anwendungen der Gentechnik vor allem in professionellen Institutionen diskutiert. Im Zentrum stehen vor allem zwei Themenkomplexe: Risikofragen und tierethische Fragen (zur Diskussion vgl. Bundesärztekammer, 1999; Referenzzentrum für Bioethik). ${ }^{14}$

Ein neues Momentum hat die Diskussion über Xenotransplantationen bekommen, als im Dezember 2018 eine Münchner Forschungsgruppe im Wissenschaftsmagazin Nature berichtete, dass es erstmals gelungen sei, Paviane mit genetisch modifizierten Schweineherzen ein halbes Jahr am Leben zu erhalten (Längin et al., 2018). Dies wurde als Durchbruch für die Forschung zu Xenotransplantationen auch am Menschen gewertet, auch wenn der Weg zu klinischen Versuchen am Menschen noch weit sei.

13 Etwa die Eurobarometer-Befragungen zur Gentechnik.

14 Siehe unter: https://www.drze.de/im-blickpunkt/organtransplantation/ethische-aspekte/?sear chterm=xenotransplantation [03.03.2021]. 
Ein alternativer Ansatz zur Lösung wird darin gesehen, künstliche Organe mithilfe von Stammzellen, sogenannten Organoiden, zu erzeugen (siehe Zenke/Bartfeld, Kap. 5). Dies ist jedoch, mehr noch als die Xenotransplantation, die immerhin bei Primaten im Versuch angewandt wird, nur eine Zukunftsvision. Gegenwärtig ist es allenfalls möglich, aus Stammzellen organspezifische Zellgebilde herzustellen. ${ }^{15}$

Obwohl nicht absehbar ist, ob und wann aus Stammzellen oder Xenotransplantationen gewonnene künstliche Organe für Menschen zur Verfügung stehen werden, wurde im TechnikRadar 2020 nach deren Akzeptanz in der Öffentlichkeit gefragt.

Abbildung 2: Einstellungen zu Xenotransplantationen und Organen aus Stammzellen zu der Frage: "Dieses Verfahren würde ich bei mir selbst akzeptieren"; in \%

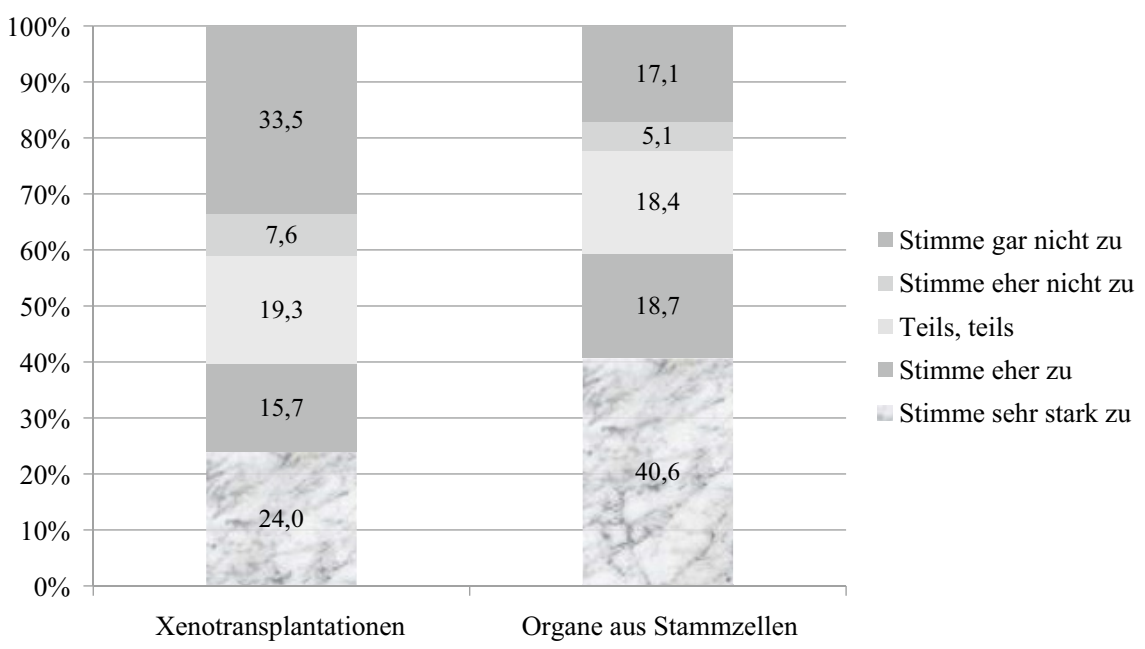

Datenbasis: TechnikRadar 2020, N=1990-1996.

In einer für das TechnikRadar durchgeführten Fokusgruppe zum Thema Organgewinnung und -spende waren Tierschutzargumente entscheidend für die Ablehnung von Xenotransplantationen. Wir haben daher im Survey gefragt, ob Tiere in jeder Hinsicht dem Menschen gleichgestellt werden sollen. Der Zusammenhang zwischen der ethischen Bewertung der Xenotransplantation und der Einschätzung, dass Tiere dem Menschen unbedingt gleichzustellen sind, war insgesamt nur mäßig stark $\left(\gamma=0,18^{* *}\right)$. Je

15 Für eine aktuelle Übersicht zum Stand der Forschung und ethischen Diskussion zu Organoiden siehe den aktuellen Themenband der IAG Gentechnologiebericht: Bartfeld et al. (2020). 
stärker Befragte der Gleichstellung von Mensch und Tier zustimmen, für umso weniger akzeptabel halten sie die Implantation eines Tierorgans bei sich selbst.

Neben den Einstellungen zu Tierrechten wirft die Übertragung tierischer Organe auf Menschen auch grundlegende Fragen nach der menschlichen Identität auf. Gerade die in Deutschland dominierenden monotheistischen Religionen betonen die prinzipielle Höherwertigkeit des Menschen. Die damit verbundene Distanz zwischen Mensch und Tier lässt eine eher kritische Haltung zur Verwendung von aus Tieren stammender Organe im eigenen Körper erwarten. Bei Betrachtung der Daten finden wir allerdings keinen nennenswerten Zusammenhang zwischen Religiosität und der Einstellung zu Xenotransplantationen.

Als unproblematischer als Xenotransplantationen werden künstliche Organe aus hES-Stammzellen angesehen. Beinahe 60 \% (59,3 \%) würden derart gewonnene Spenderorgane bei sich akzeptieren, wobei sich höher gebildete Befragte noch etwas aufgeschlossener zeigen (66,1\%) als Befragte mit niedrigem Bildungsstand (53,0 \%). 61,8 \% der Befragten halten sie für ethisch akzeptabel und über 40,0\% stimmen hier sogar sehr stark zu. Auf der anderen Seite hält nur jeder Fünfte (18,5 \%) die Transplantation von im Labor gezüchteten, künstlichen Organen für ethisch inakzeptabel. Auch hier gibt es keinen statistisch nennenswerten Zusammenhang mit der Religiosität $(\gamma=-0,08)$.

Zwar bleibt das Spenderorgan vom Menschen für fast zwei Drittel der Befragten $(61,9 \%)$ nach wie vor die erste Präferenz. Dennoch ist die Zustimmung insbesondere zu künstlichen Organen aus Stammzellen überraschend hoch. Organe aus dieser Quelle wären immerhin für ein Drittel (34,3 \%) die erste Wahl. Organe aus Xenotransplantationen finden dagegen nur geringe Zustimmung. Nur knapp $4 \%$ der Befragten präferieren in Tieren gezüchtete Organe.

\subsubsection{Gentherapien}

Anders als Organtransplantationen zielen Gentherapien darauf ab, Krankheiten im Organismus selbst zu heilen (vgl. Fehse/Domasch, 2011; Fehse/Domasch, 2015; siehe auch Fehse, Kap. 6). Anders als Xenotransplantationen und neue Organe sind Gentherapien über das Laborstadium längst hinausgewachsen (Fehse/Domasch, 2015). Neue Impulse bekam die Diskussion über Gentherapien mit der Entdeckung von CRISPR/Cas9, einer Methode, die auch im medizinischen Bereich gezieltere Eingriffe in das Erbgut gestattet als herkömmliche Verfahren und eine intensive Debatte in der Wissenschaft über die ethisch gebotenen Grenzen der Gentherapie auslöste. Diese Diskussion fand vor allem in den international führenden Wissenschaftszeitschriften Nature und Science statt (eine systematische Analyse der Diskussion findet sich bei Reich et al., 2015). 
Prinzipiell werden drei Arten von Gentherapien unterschieden: die somatische Gentherapie am geborenen Menschen, die somatische Gentherapie am Embryo und die Keimbahntherapie. Die somatische Gentherapie zielt darauf ab, bei einer auf einen Gendefekt zurückzuführenden Erkrankung gezielte Veränderungen am Erbgut der Körperzellen (somatische Zellen) eines Patienten vorzunehmen, um diese Erkrankung zu heilen (vgl. Fehse/Domasch, 2015). Wirkungsvoller ist eine Gentherapie dann, wenn sie bereits an einem Embryo durchgeführt wird - sie gilt als umso effektiver je früher der therapeutische Eingriff ansetzt. Gentherapien am Embryo sind allerdings mit dem Risiko verbunden, dass die genetische Veränderung an Nachkommen weitergegeben wird. Zielt die Gentherapie auf die Änderung somatischer Zellen des Embryos ab und nicht auf die Keimzellen, spricht man von somatischer Gentherapie beim Embryo. Ist hingegen eine Weitergabe der Veränderung über die Keimzellen (auch Keimbahnzellen) Ziel des Eingriffs, wird dieser als Keimbahntherapie bezeichnet.

Mit dem deutschen Biotech-Survey von 1997 wurden die Einstellungen zur Gentechnik differenziert erhoben (Hampel/Renn, 1999: 32 f.). Die Gentherapie, die damals eher ein Versprechen für die Zukunft als Realität war, erzielte von allen Anwendungen der Gentechnik die größte Zustimmung. International wurden die Einstellungen zur Gentherapie vor allem in einigen Eurobarometerstudien, insbesondere im Eurobarometer 64.3 von 2005 und im Eurobarometer 73.1 von 2010 thematisiert (Hampel, 2011).

Eine Reihe empirischer Studien erlaubt es uns, den Expertendiskurs in den Fachzeitschriften durch die Perspektive der Öffentlichkeit auf Genome-Editing zu ergänzen. Studien liegen aus Europa und den USA, aber auch aus China vor, wobei einschränkend hervorgehoben werden muss, dass nur zwei dieser Studien - der STAT \& Harvard T.H. Chan Studie (2016) und der Studie des Pew Research Centers (Funk/Hefferon, 2018) Zufallsstichproben zugrunde liegen. Andere Studien basieren lediglich auf mehr oder weniger methodisch kontrollierten Online-Stichproben. Dies muss bei der Interpretation berücksichtigt werden.

Wie auch bei anderen Anwendungen der Gentechnik hängt die Akzeptanz der Gentherapie wesentlich vom Kontext ihrer Anwendung und Zielsetzungen ab. Von zentraler Bedeutung sind dabei die folgenden Gesichtspunkte: dient die Gentherapie Therapie- oder Enhancementzwecken, wird sie am (einwilligungsfähigen) Erwachsenen oder am Embryo durchgeführt und bleiben die Veränderungen auf das behandelte Individuum beschränkt oder werden sie an Nachkommen weitergegeben? Die Studie von Gaskell et al. (2017) zeigt deutlich, dass die therapeutische Behandlung von Erwachsenen auf große Zustimmung stößt, wohingegen pränatale Eingriffe zur Verbesserung 
des Genoms (Enhancement) einhellig abgelehnt werden. ${ }^{16}$ Pränatale therapeutische Eingriffe werden zwar, wie therapeutische Eingriffe bei Erwachsenen, mehrheitlich befürwortet, die Bandbreite der Urteile ist aber wesentlich größer als bei Erwachsenen.

Amerikanische Studien, etwa von Scheufele (2017) und dem Pew Institute (Funk/ Hefferon, 2018), finden eine verbreitete Zustimmung zu Genome-Editing in den USA. Bei therapeutischen Anwendungen liegt die Zustimmung bei über $60 \%$; wenn eine Krankheit unmittelbar nach der Geburt einzutreten droht, sogar bei 72 \% (Funk/Hefferon, 2018).

Die US-Studien haben auch untersucht, welche sozialen Merkmale hinsichtlich der Einschätzung des Genome-Editing variieren. Scheufele und das Pew Institute kommen zu den gleichen Ergebnissen, wonach religiöse Menschen dem therapeutischen Genome-Editing kritischer gegenüberstehen als weniger religiöse, und Menschen mit hohem Wissensniveau aufgeschlossener sind als Menschen mit niedrigem.

Die vorliegenden empirischen Studien zeigen, dass Gentherapien unterschiedlich wahrgenommen werden, je nachdem in welchem Kontext man sie anwendet. Dabei ist insbesondere bei der Therapie genetisch bedingter Erkrankungen ein Dilemma zu beobachten. Um wirksam zu werden, ist es angebracht, Gentherapien möglichst bereits im embryonalen Stadium anzuwenden, am besten, bevor sich die Zellen ausdifferenziert haben. Die Akzeptanz für Gentherapien ist aber bei Erwachsenen höher. Je weniger ausdifferenziert die Zellen sind, umso größer ist aber auch das Risiko, dass die genetische Veränderung auch die Keimbahn betrifft. Es handelt sich hierbei um die kritische Abgrenzung zwischen somatischer und Keimbahntherapie, die auch das Erbgut verändert.

Auch wenn die Keimbahntherapie in Deutschland verboten ist und die Zeit von Wissenschaftlern für (noch?) nicht reif für die Keimbahntherapie gehalten wird, haben wir uns entschlossen, im TechnikRadar 2020 die gesamte Bandbreite der Gentherapie zu erfassen: von der somatischen Gentherapie am Erwachsenen über die somatische Gentherapie am Embryo bis hin zur Keimbahntherapie, um damit für zukünftige Debatten Informationen bereitstellen zu können.

Wir finden im TechnikRadar eine klare Abstufung der Akzeptanz von der somatischen Gentherapie beim Erwachsenen, die von 70,0 \% der Deutschen befürwortet wird, über die somatische Gentherapie am Embryo, bei der die Zustimmung auf 36,0 \% zurückgeht, bis zur Keimbahntherapie am Embryo, die nur noch von fast jedem Fünften $(18,8 \%)$ befürwortet wird. Während die somatische Gentherapie am Erwachsenen nur

16 Diese Studie bezog sich ausschließlich auf Varianten der Gentherapie. Die Keimbahntherapie war nicht Gegenstand der Studie. 
selten Ablehnung hervorruft (9,3\%), sind es bei der somatischen Gentherapie am Embryo bereits $34,5 \%$ und - überraschend gering - bei der Keimbahntherapie lediglich 49,3\%. Um Framing-Effekte untersuchen zu können, das heißt, um zu überprüfen, welche Auswirkungen unterschiedliche Perspektiven auf das Antwortverhalten haben, wurde bei der Befragung eine Split-Half-Methode eingesetzt: Dabei wurden die Einstellungen zur Keimbahntherapie auf unterschiedliche Weise formuliert und beide Varianten bei der Befragung zufällig eingesetzt.

Abbildung 3: Einstellungen zu Varianten der Gentherapie

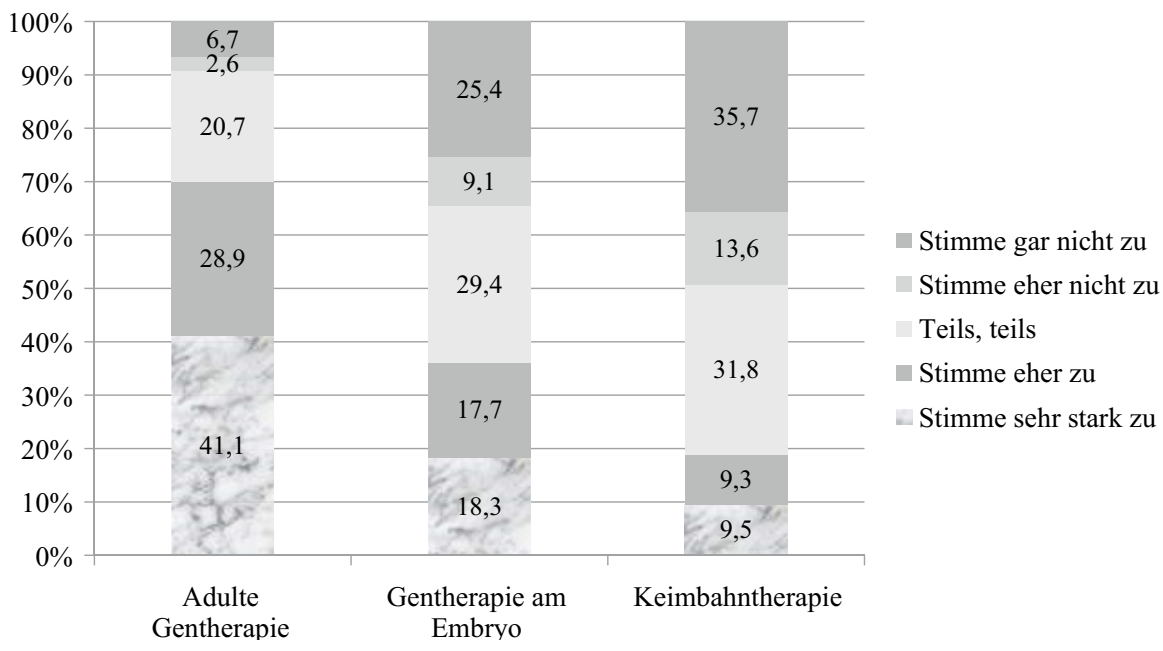

Datenbasis: TechnikRadar 2020, N=1990-1996.

Insgesamt ist die Gentherapie am Erwachsenen zu einer weitgehend akzeptierten Angelegenheit geworden. Anders sieht es bei der somatischen Gentherapie am Embryo aus, die gleichermaßen auf Zustimmung wie auf Ablehnung stößt. Immerhin ein Drittel $(36,0 \%)$ befürwortet diese Anwendung. Auffällig ist aber, dass, anders als bei der Gentherapie am Erwachsenen, der Anteil entschieden ablehnender Urteile deutlich größer ist (25,4 \% gegenüber 6,7 \% bei der Gentherapie am Erwachsenen). Wenn die genetische Veränderung an Nachkommen weitergegeben wird, also bei der Keimbahntherapie, dominiert dagegen eindeutig die Ablehnung. Zwar befürwortet nur gut jeder Sechste $(18,8 \%)$ diese Therapieform, aber ein starkes Drittel (35,7 \%) lehnt sie entschieden ab.

Bei Variante A wurde der medizinische Langzeiteffekt dahingehend betont, dass erbliche Krankheiten nicht mehr an Nachkommen weitergegeben werden. Diese kon- 
sequentialistische Frageformulierung führte zu 47,4 \% Zustimmung und 25,9 \% Ablehnung.

Fragevariante B lautete: „Eine solche Therapie am Embryo finde ich auch dann gut, selbst wenn die genetische Veränderung an künftige Generationen weitergegeben wird." Diese Variante rief bei 18,8 \% Zustimmung und bei 49,3 \% Ablehnung hervor. ${ }^{17}$ In den oben zitierten amerikanischen Studien war ein wichtiger Prädiktor für die Einstellungen zur Gentherapie die Religiosität. Je religiöser eine Person war, desto wahrscheinlicher war die Ablehnung der Gentherapie. In Deutschland gibt es diesen Zusammenhang nicht. Das gilt sowohl für die somatische Gentherapie am Erwachsenen $(\gamma=0,03)$, die somatische Gentherapie am Embryo $\left(\gamma=0,05^{*}\right)$ als auch die Keimbahntherapie $(\gamma=0,06)$. Selbst von den sehr Religiösen lehnen nur $11,4 \%$ die Gentherapie am Erwachsenen ab. Ähnlich der Religiosität hat in Deutschland auch der Bildungsstand so gut wie keinen Einfluss auf Einstellungen zur Gentherapie.

Die Gentherapie und allen voran die somatische Gentherapie am Erwachsenen ist zu einer weitgehend akzeptierten Heilungsmethode geworden. Die somatische Gentherapie am Embryo wird durchaus kontrovers diskutiert und von einem Viertel der Deutschen abgelehnt. Stärker noch ist die Ablehnung der Keimbahntherapie.

Die vergleichsweise hohe Zustimmung zur Keimbahntherapie, wenn die Frageformulierung nicht auf die Weitergabe genetischer Veränderungen an Folgegenerationen, sondern auf die Vermeidung von Erkrankungen in Folgegenerationen abzielt, wirft die Frage auf, ob nicht bei entsprechenden Therapieerfolgen ein gesellschaftlicher Druck hin zu einer Liberalisierung der Keimbahntherapie entstehen könnte.

\subsection{Fazit}

Bereits in den 1990er Jahren durchgeführte empirische Studien kamen zu dem Ergebnis, dass gentechnische Anwendungen sehr unterschiedlich wahrgenommen werden. Während die meisten medizinischen Anwendungen der Gentechnik auf große Zustimmung stießen, war die Reaktion auf die Grüne Gentechnik von Ablehnung geprägt, nicht nur in Deutschland.

Dieses Muster ist auch gegenwärtig noch zu beobachten, auch wenn sich die Gentechnik seit den 1990er Jahren sehr stark verändert hat. Einerseits wurden neue Ver-

17 Weitergehende Analysen der Daten haben gezeigt, dass sich ein Teil der Befragten nicht bewusst war, dass eine Therapie, die verhindert, dass eine genetisch verursachte Krankheit an Nachkommen weitervererbt wird, eine Keimbahntherapie ist. Nur so ist zu erklären, dass Befragte gleichzeitig die Keimbahntherapie am Embryo befürworteten, aber die ethisch wesentlich unproblematischere somatische Gentherapie am Erwachsenen ablehnten. 
fahren entwickelt, CRISPR/Cas9 ist das bekannteste, die die Präzision gentechnischer Eingriffe erhöht haben, anderseits wurden, nicht zuletzt als Folge der Erweiterung der technischen Möglichkeiten, neue Anwendungen entwickelt, die neue Diskussionen auslösten.

Konstant ist die Ablehnung der Grünen Gentechnik. Nach wie vor wird ihre Wahrnehmung von einer fehlenden Nutzenperspektive geprägt. Ohne wahrgenommenen Nutzen werden wahrgenommene Risiken zu einem K.-o.-Kriterium.

Die erstmals erhobene Wahrnehmung der Mutagenesezüchtung, die überraschenderweise noch stärker abgelehnt wird als die Grüne Gentechnik, zeigt, dass die Ablehnung nicht gentechnikspezifisch ist. Die genauen Gründe dafür können hier nur angerissen werden. Zu vermuten ist, dass die konventionelle Landwirtschaft kulturell in die Defensive geraten ist. Ein Blick auf die Lebensmittelwerbung zeigt, dass die hier propagierten Qualitätskriterien wie Natürlichkeit und Tradition nur schwer mit einer modernen High-Tech-Landwirtschaft zu vereinbaren sind.

Ist die Wahrnehmung der Grünen Gentechnik in der Öffentlichkeit von einer fehlenden Nutzenperspektive geprägt, wird mit dem Einsatz von Laborfleisch die Lösung mehrerer mit der konventionellen Fleischproduktion verbundener Probleme versprochen, vom Ressourcenverbrauch über den Ausstoß von Treibhausgasen bis hin zu ethischen Fragen der Tiernutzung. Aber auch hier reagiert die deutsche Öffentlichkeit mehrheitlich ablehnend. Die unterstellten ethischen Vorteile werden nicht wahrgenommen und fast die Hälfte hält Laborfleisch für risikoreicher als herkömmliches Fleisch, Dass eine weitere Entfremdung der Menschen von der Produktion von Lebensmitteln befürchtet wird, spricht für die These der Entfremdung von der modernen Landwirtschaft. Anders als bei der Grünen Gentechnik, für die es kein erkennbares Befürworterprofil gibt, finden wir bei Laborfleisch eine deutlich überproportionale Zustimmung bei Jüngeren mit höheren Bildungsabschlüssen. Möglicherweise entwickeln sich hier Nischenmärkte, die gegenüber dieser Innovation aufgeschlossen sind.

Das alte Bild von abgelehnten Anwendungen der Grünen Gentechnik und akzeptierten medizinischen Anwendungen der Gentechnik muss differenziert werden. Die Gentherapie hat sich zu einer allgemein anerkannten Methode entwickelt, jedenfalls dann, wenn man sie am (zustimmungsfähigen) Erwachsenen durchführt. Anwendungen am Embryo und mehr noch die Keimbahntherapie stoßen auf eine geringe Zustimmung oder werden deutlich abgelehnt. Zurückhaltend werden auch im Labor gezüchtete Organe und vor allem Xenotransplantationen bewertet. Auch wenn Organe von menschlichen Spendern bevorzugt werden, würde die Mehrheit zumindest im Labor gezüchtete Organe bei sich akzeptieren. Die nur geringe Zustimmung zu Xenotransplantationen 
spricht dafür, dass es sich dabei aus gegenwärtiger Sicht allenfalls um eine Übergangstechnologie handelt.

Die beobachtete Ausdifferenzierung der Einstellungen zu den verschiedenen Anwendungen gentechnischer Methoden zeigt, dass nicht so sehr die Gentechnik im Vordergrund steht, sondern das, was damit gemacht wird. Eine Gentechnikkommunikation, die sich als Wissenschaftsmarketing oder reine Wissenschaftskommunikation im Sinne einer Vermittlung von Faktenwissen alleine versteht, zielt damit am gesellschaftlichen Diskursbedarf vorbei.

\subsection{Literaturverzeichnis}

acatech/Körber-Stiftung (Hrsg.) (2020): TechnikRadar 2020. Was die Deutschen über Technik denken. Schwerpunkt: Bioökonomie. acatech/Körber-Stiftung, München/Hamburg. Unter: https://www. acatech.de/publikation/technik-radar-2020/ [03.03.2021].

Bartfeld, S. et al. (2020): Organoide. Ihre Bedeutung für Forschung, Medizin und Gesellschaft. Nomos, Baden-Baden. DOI: 10.5771/9783748908326.

Böhm, I. et al. (2018): Visions of in vitro meat among experts and stakeholders. In: Nanoethics 12(3): 211-224. DOI: 10.1007/s11569-018-0330-0.

Bryant, C./Barnett, J. (2018): Consumer acceptance of cultured meat: A systematic review. In: Meat Science 143: 8-17. DOI: 10.1016/j.meatsci.2018.04.008.

Bundesärztekammer (1999): Stellungnahme des Wissenschaftlichen Beirats der Bundesärztekammer zur Xenotransplantation. In: Deutsches Ärzteblatt 96(28-29): A1920-A1926.

De Cheveigné, S. et al. (2006): „Spare parts for human bodies“. In: Gaskell, G./Bauer, M. W. (Hrsg.): Genomics and society. Legal, ethical and social dimensions. Earthscan, London: 28-43.

Durant, J. et al. (Hrsg.) (1998): Biotechnology in the public sphere. A European sourcebook. Science Museum Press, London.

$\mathrm{EU}=$ European Commission (2005): Special Eurobarometer 225: Social values, science and technology. Brüssel. Unter: https://data.europa.eu/data/datasets/s448_63_1_ebs225?locale=en [03.03.2021].

Fehse, B./Domasch, S. (Hrsg.) (2011): Gentherapie in Deutschland. Eine interdisziplinäre Bestandsaufnahme. Forschungsberichte der Interdisziplinären Arbeitsgruppen der Berlin-Brandenburgischen Akademie der Wissenschaften, Band 27. 2., aktualisierte und erweiterte Auflage. Forum W, Dornburg.

Fehse, B./Domasch, S. (2015): Themenbereich somatische Gentherapie: Translationale und klinische Forschung. In: Müller-Röber, B. et al. (Hrsg.): Dritter Gentechnologiebericht. Analyse einer Hochtechnologie. Forschungsberichte der Interdisziplinären Arbeitsgruppen der Berlin-Brandenburgischen Akademie der Wissenschaften, Band 32. Baden-Baden: Nomos: 210-307. 
Funk, C./Hefferon, M. (2018): Public views of gene editing for babies depend on how it would be used. Pew Research Center, Washington, D.C. Unter: https://www.pewresearch.org/science/2018/07/26/ public-views-of-gene-editing-for-babies-depend-on-how-it-would-be-used/ [03.03.2021].

Gaskell, G./Bauer, M. W. (Hrsg.) (2001): Biotechnology 1996-2000. The years of controversy. Science Museum Press, London.

Gaskell, G. et al. (2004): GM foods and the misperception of risk perception. In: Risk Analysis 24(1): 185-194. DOI: 10.1111/j.0272-4332.2004.00421.x.

Gaskell, G. et al. (2017): Public views on gene editing and its uses. In: Nature Biotechnology 35(11): 1021-1023. DOI: 10.1038/nbt.3958.

Hampel, J. (2011): Wahrnehmung und Bewertung der Gentherapie in der deutschen Bevölkerung. In: Fehse, B./Domasch, S. (Hrsg): Gentherapie in Deutschland. Eine interdisziplinäre Bestandsaufnahme. Forschungsberichte der Interdisziplinären Arbeitsgruppen der Berlin-Brandenburgischen Akademie der Wissenschaften, Band 27. 2., aktualisierte und erweiterte Auflage. Forum W, Dornburg: 227-255.

Hampel, J./Renn, O. (Hrsg.) (1999): Gentechnik in der Öffentlichkeit. Wahrnehmung und Bewertung einer umstrittenen Technologie. Campus, Frankfurt am Main.

Hampel, J./Zwick, M. M. (2016): Wahrnehmung, Bewertung und die Akzeptabilität von Technik in Deutschland. Die Problematik der Erfassung von Technikeinstellungen am Beispiel von externer Technik und Gentechnik. In: TATuP 25(1): 24-38.

Jinek, M. et al. (2012): A programmable dual-RNA-guided DNA endonuclease in adaptive bacterial immunity. In: Science 337/6096: 816-821. DOI: 10.1126/science.1225829.

Kronberger, N. et al. (2013): How natural is "more natural“? The role of method, type of transfer, and familiarity for public perceptions of cisgenic and transgenic modification. In: Science Communication 36(1): 106-130. DOI: 10.1177/1075547013500773.

Laestadius, L. (2015): Public perceptions of the ethics of in-vitro meat: determining an appropriate course of action. In: Journal of Agricultural and Environmental Ethics 28(5): 991-1009. DOI: 10.1007/s10806-015-9573-8.

Längin, M. et al. (2018): Consistent success in life-supporting porcine cardiac xenotransplantation. In: Nature 564(7736): 430-433. DOI: 10.1038/s41586-018-0765-z.

Petermann, T./Sauter, A. (1999): TA-Monitoring „Xenotransplantation“. Sachstandsbericht. Arbeitsbericht Nr. 64. Büro für Technikfolgen-Abschätzung beim Deutschen Bundestag, Berlin. Unter: https://www.tab-beim-bundestag.de/de/pdf/publikationen/berichte/TAB-Arbeitsbericht-ab064. $\operatorname{pdf}[03.03 .2021]$.

Post, M. J. (2014): Cultured beef: medical technology to produce food. In: Journal of the Science of Food and Agriculture 94(6): 1039-1041. DOI: 10.1002/jsfa.6474. 
Reich, J. et al. (2015): Genomchirurgie beim Menschen. Zur verantwortlichen Bewertung einer neuen Technologie. Analyse der Interdisziplinären Arbeitsgruppe Gentechnologiebericht, Berlin-Brandenburgischen Akademie der Wissenschaften, Berlin. Unter: https://www.gentechnologiebericht. de/fileadmin/user_upload/Webseitendateien/Publikationen/deutsch_Genomchirurgie-beimMenschen_2015.pdf [03.03.2021].

Scheufele, D. A. et al. (2017): U.S. attitudes on human genome editing. In: Science 357(6351): 553-554. DOI: $10.1126 /$ science.aan3708.

Schicktanz, S. (2000): Aus der Geschichte lernen? Die Entwicklung der Idee der Xenotransplantation und Auswirkungen auf das heutige Verständnis. In: Engels, E.-M. et al. (Hrsg.): Neue Perspektiven der Transplantationsmedizin im interdisziplinären Dialog. Nomos, Baden-Baden: 239-256.

STAT/Harvard T. H. Chan School of Public Health (2016): The public and genetic editing, testing, and therapy. Harvard T.H. Chan School of Public Health, Boston. Unter: https://cdn1.sph.harvard. edu/wp-content/uploads/sites/94/2016/01/STAT-Harvard-Poll-Jan-2016-Genetic-Technology.pdf [03.03.2021].

Torgersen, H. et al. (2002): Promise, problems and proxies: twenty-five years of debate and regulation in Europe. In: Bauer, M. W./Gaskell, G. (Hrsg.): Biotechnology. The making of a global controversy. Cambridge University Press, Cambridge: 21-94.

Woll, S. (2019): On visions and promises - ethical aspects of in vitro meat. In: Emerging Topics in Life Sciences 3(6): 753-758. DOI: 10.1042/ETLS20190108. 